\title{
Making Sense of Illustrated Handwritten Archives
}

MAKING SENSE realizes a technologically advanced and user-friendly digital infrastructure to open up, enrich and connect illustrated handwritten archives. It combines both image and textual recognition, and allows for an integrated study of underexplored digitized scientific collections. This approach is applicable across the cultural heritage domain and is demonstrated using a 17,000 page account of the exploration of the Indonesian Archipelago between 1820 and 1850 ("Natuurkundige Commissie voor Nederlands-Indië"). This poster provides a project overview, presents the infrastructure's basic layout and sketches its realization in the period 2016-2020. Funding for this project is provided by the Netherlands Organization for Scientific Research (NWO) and BRILL publishers.

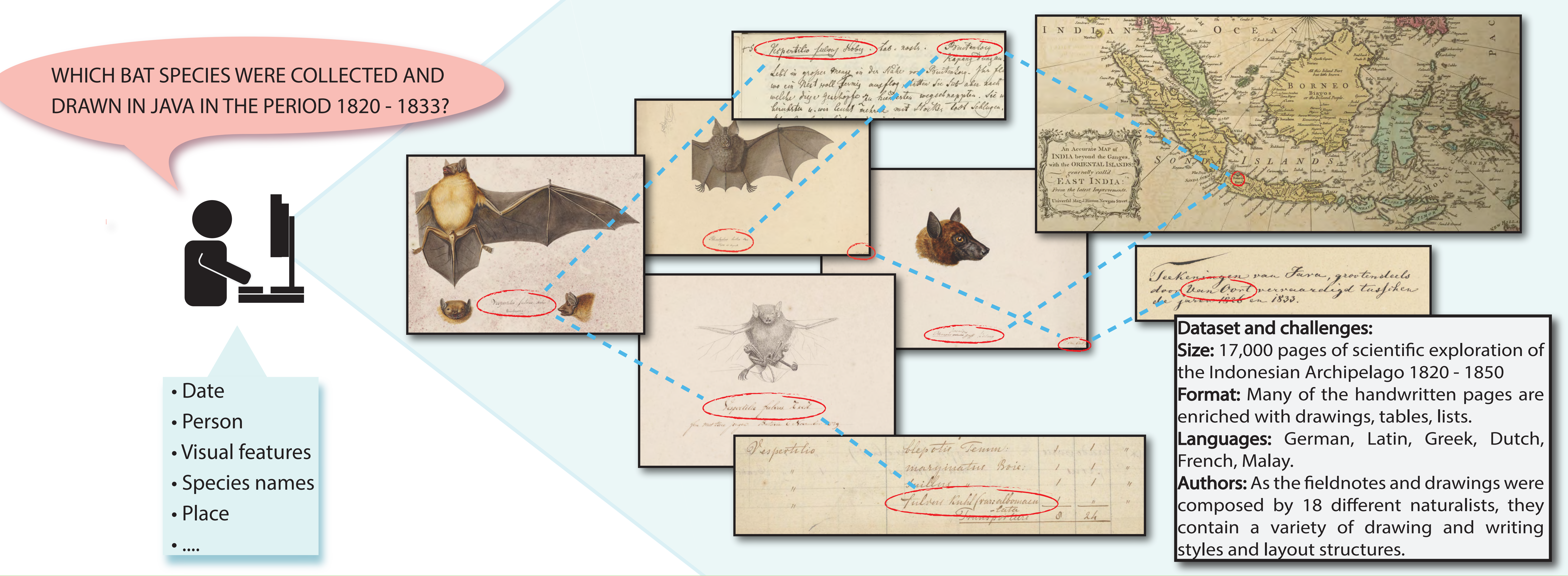

Lexicon \& Ontologies Identify and construct vocabularies and ontologies that can be used as background knowledge and the formal representation of these resources.

\section{Preprocessing Apply noise removal, binarization and normalization on page images. \\ Layout Analysis Extract regions of interests (ROI) from document images through a geometrical and logical analysis.}

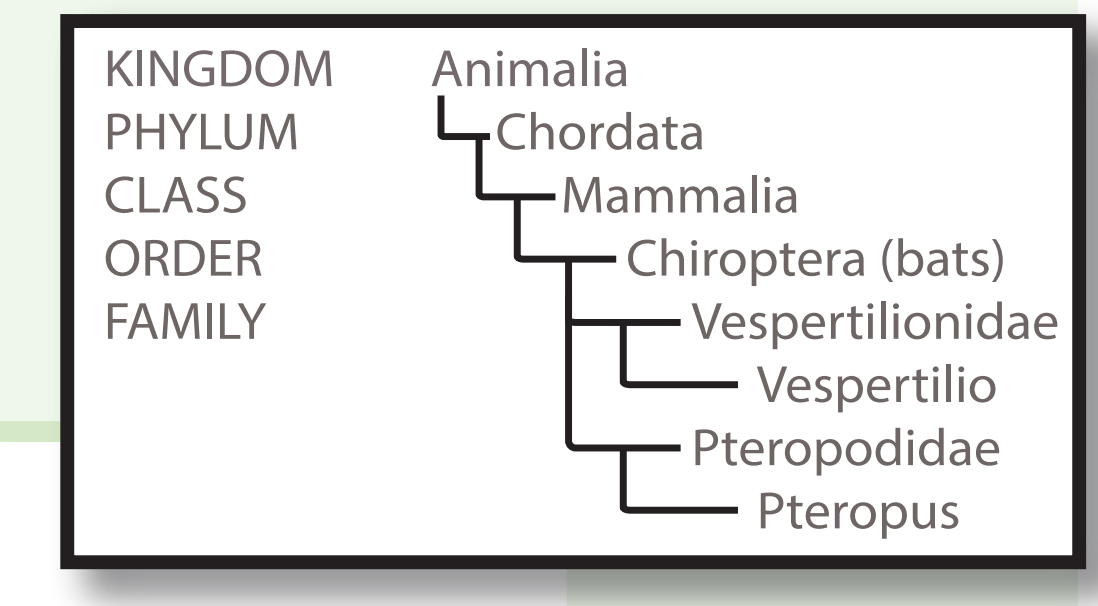

Recognize page segments and form hypotheses about their content. The historical collec-

Text and Picture Recognition tion contains text, drawings of animals and plants and tables with numerical data. The challenge is to extract as much information from a scanned image. We will use layout analysis and segmentation to arrive at text and object classification using (deep) machine learning. Already the low-level problem of segmentation requires knowledge.

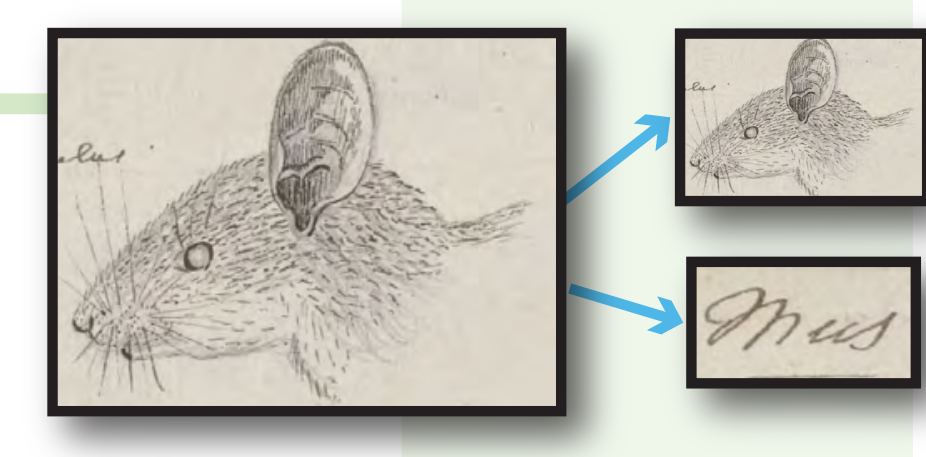

Select background knowledge that can be used to improve the accuracy of the recognition

Integration process. Develop algorithms based on probabilistic logic programming to integrate background knowledge, candidate words and candidate images.

\section{Outreach} specimen collection databases as well as other cultural heritage resources.
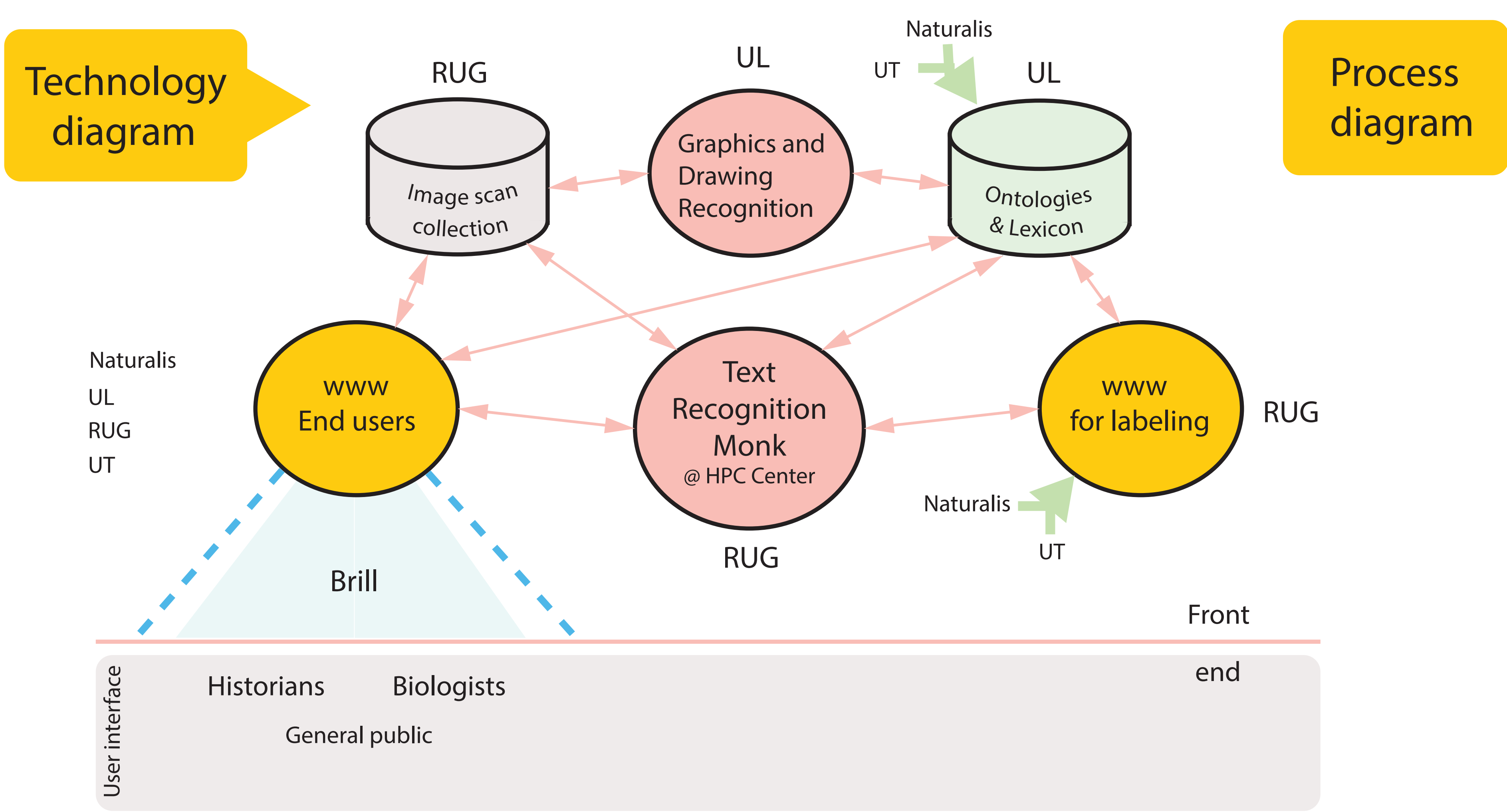
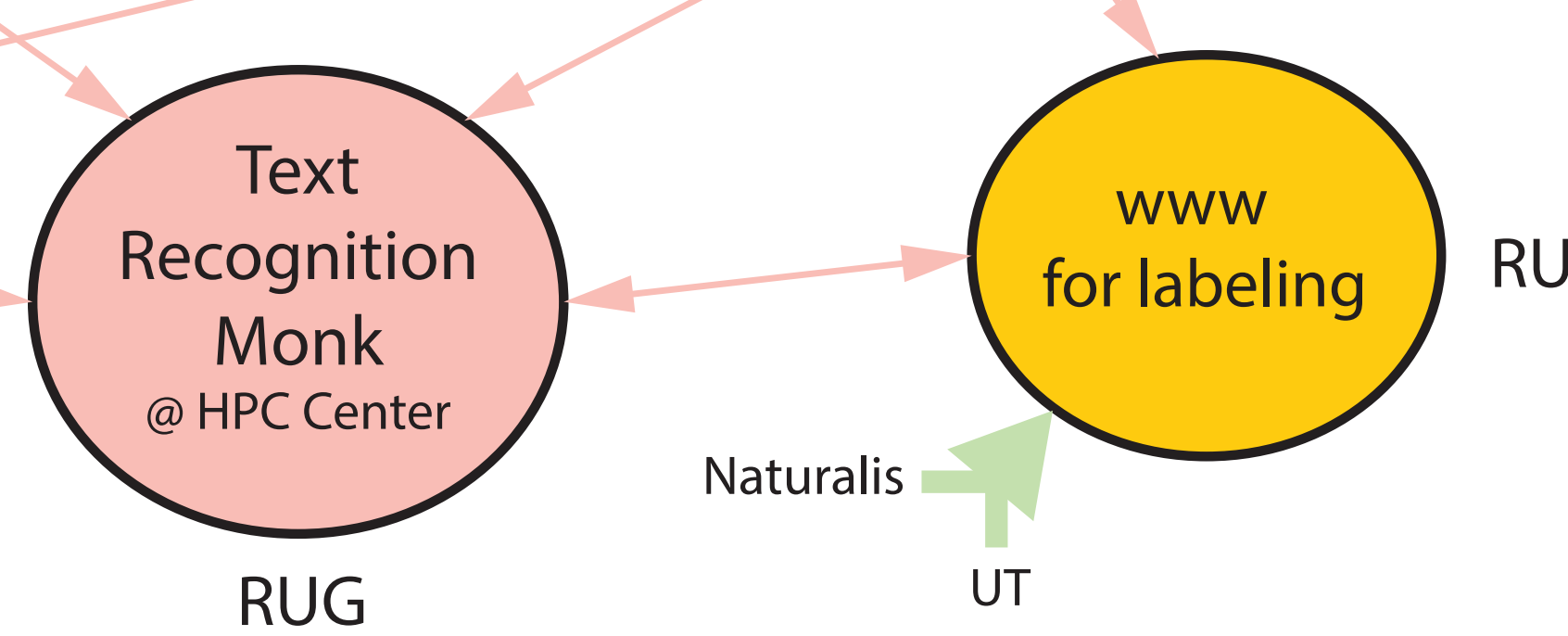

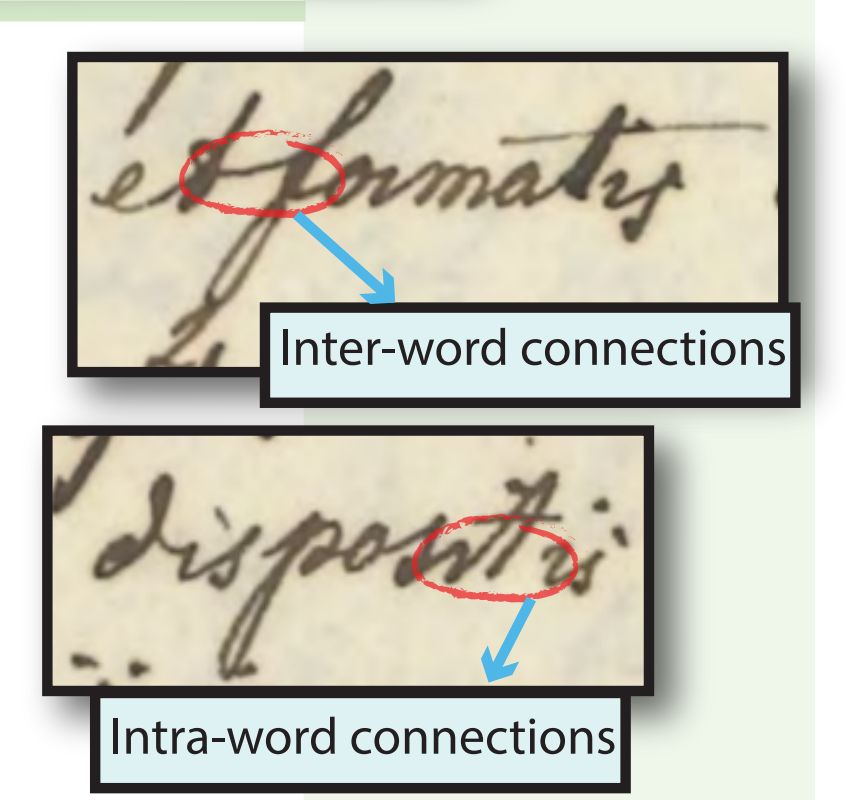

